\title{
Strain analysis of InGaN/GaN multi quantum well LED structures
}

\author{
S. Şebnem Çetin ${ }^{* 1,2}$, M. Kemal Öztürk ${ }^{1,2}$, S. Özçelik ${ }^{1,2}$, and E. Özbay ${ }^{3}$ \\ ${ }^{1}$ Department of Physics, Science Faculty, Gazi University, 06500, Ankara, Turkey \\ ${ }^{2}$ Photonics Application and Research Center, Gazi University, 06500, Ankara, Turkey \\ ${ }^{3}$ Nanotechnology Research Center, Department of Physics, Department of Electrical and Electronics \\ Engineering, Bilkent, 06800, Ankara, Turkey
}

Received 27 May 2011, revised 30 April 2012, accepted 21 May 2012 Published online 22 June 2012

\begin{abstract}
Key words InGaN, GaN, multi quantum well, high resolution x-ray diffraction, strain-stress analysis, light emitted diode.

Five period InGaN/GaN multi quantum well (MQW) light emitting diode (LED) structures were grown by a metalorganic chemical vapor deposition (MOCVD) system on $c$-plane sapphire. The structural characteristics as a strain-stress analysis of hexagonal epilayers MQWs were determined by using nondestructive high resolution x-ray diffraction (HRXRD) in detail. The strain/stress analysis in AlN, GaN, and InGaN thin films with a variation of the In molar fraction in the InGaN well layers was conducted based on the precise measurement of the lattice parameters. The $a$ - and $c$-lattice parameters of the structures were calculated from the peak positions obtained by rocking the theta axis at the vicinity of the symmetric and asymmetric plane reflection angles, followed by the in-plane and out-of-plane strains. The biaxial and hydrostatic components of the strain were extracted from the obtained a- and c-direction strains values.
\end{abstract}

(C) 2012 WILEY-VCH Verlag GmbH \& Co. KGaA, Weinheim

\section{Introduction}

Group III-nitride based semiconductors have gained much interest in optical device applications in the industry for the visible spectral regime in the past decade [1-4]. Especially, high-brightness light emitting diodes (LEDs), operating at short visible wavelengths, violet, blue, and green, were made possible by the development of metalorganic chemical vapor deposition and metalorganic vapor phase epitaxy growth techniques [5,6]. Currently, it is still difficult to obtain high-quality LEDs because of the large lattice mismatch and the difference in the thermal expansion coefficients (TECs) between the films and substrate [7]. The reason for this is the difference that results in high level strain, stress, and mosaic defects, such as screw and edge type dislocations, lateral and vertical coherence lengths, as well as tilts and twist angles [8,9]. To produce a quality LED, its characterizing parameters (compound, thickness, growth temperature, etc.) are basically conducted. Therefore, the studies made on strainstress are very important for resolving the defect structure that appeared in LED growth. Recently, a number of discussions on strain-stress over the different problems were given in the literature [10-14]. Kisielowski et al. [10] firstly presented that the superposition of a hydrostatic and a biaxial strain field determines the observed stress in GaN thin films. Precise information on the composition of the strained layer in the alloy semiconductors can be obtained by HRXRD measurements using well established simulation procedure including the Takagi-Taupin formulation of dynamic X-ray theory [15]. The lattice parameters of the compounds can also be evaluated from the positions of the diffraction peaks measured by using the HRXRD technique. In addition, the relaxed lattice parameters have been calculated from the obtained experimental values of the lattice parameters with the help of the Poisson coefficient $[10,16,17]$.

The hydrostatic pressures affect the positions of the nitrogen vacancy levels in $\mathrm{GaN}$ or $\mathrm{GaN}$ related compounds since the lattice constant is changed [18]. It can be said that the hydrostatic strain is also affected by the presence of point defects such as impurities and vacancies that alter the lattice parameters. The strain can be compressive

\footnotetext{
*Corresponding author: e-mail: cetins @ gazi.edu.tr
} 


\begin{tabular}{|c|c|c|c|}
\hline \multicolumn{4}{|c|}{ p-GaN $160 \mathrm{~nm}$} \\
\hline InGaN/GaN 5xQW & A & B & $\mathrm{C}$ \\
\hline $\begin{array}{l}\text { TMIn pressure in bubbler } \\
\text { (mbar) }\end{array}$ & 600 & 600 & 1000 \\
\hline $\mathrm{T}_{\mathrm{g}}\left({ }^{\circ} \mathrm{C}\right)$ & 720 & 710 & 710 \\
\hline \multicolumn{4}{|c|}{$\mathrm{N}^{+} \mathrm{GaN} \sim 1600 \mathrm{~nm}$} \\
\hline \multicolumn{4}{|c|}{ GaN Buffer Layer $340 \mathrm{~nm}$} \\
\hline \multicolumn{4}{|c|}{ HT-AlN Nucleation $400 \mathrm{~nm}$} \\
\hline \multicolumn{4}{|c|}{ LT-AlN Nucleation $10 \mathrm{~nm}$} \\
\hline \multicolumn{4}{|c|}{ Sapphire Substrate $330 \mu \mathrm{m}$} \\
\hline
\end{tabular}

Fig. 1 The five period $\mathrm{InGaN} / \mathrm{GaN}$ MQW structures (A, B, and $\mathrm{C}$ ).

or expansive depending on the size of the point defects. The biaxial strain originates from the lattice mismatch between the substrates and the grown epitaxial layer with different TECs $[10,11,19]$. In addition, the stress can be caused by lattice mismatch, island coalescence, grain growth, or surface stress [20-22].

In the present study, the three $5 x\left(\operatorname{In}_{x} \mathrm{Ga}_{1-x} \mathrm{~N} / \mathrm{GaN}\right) \mathrm{MQW}$ LED structures were grown by MOCVD. The values of the lattice spacing of the samples were calculated from Bragg law by using the peak positions of symmetric and asymmetric reflection obtained from HRXRD. Using the lattice parameters determined with lattice spacing, the strain, a- and c-direction strains, hydrostatic strain, biaxial strain, and biaxial stress values were calculated by using the method described in $[10,11]$ and were discussed with their reasons in detail.

\section{Experimental}

Three samples with five period-InGaN/GaN MQW LED structures were grown by MOCVD on $c$-plane (00.1)faced sapphire substrates with a $10 \mathrm{~nm}$ low-temperature (LT) AlN nucleation layer. Hydrogen was used as the carrier gas for trimethylgallium (TMGa), trimethylaluminum (TMAl), and ammonia $\left(\mathrm{NH}_{3}\right.$ ), which are used as source compounds. During the growth, the reactor pressure was kept at 200 mbar. A $10 \mathrm{~nm}$ AlN nucleation layer and $400 \mathrm{~nm}$ AlN buffer layer were deposited at $840^{\circ} \mathrm{C}$ and $1150{ }^{\circ} \mathrm{C}$, respectively. A $340 \mathrm{~nm}$ GaN buffer layer was grown at $1080^{\circ} \mathrm{C}$. A thick n-type GaN:Si layer with a thickness of $1600 \mathrm{~nm}$ was grown on the GaN buffer layer for all of the samples. Quantum well layers were formed, such that each $2 \mathrm{~nm} \operatorname{In}_{x} \mathrm{Ga}_{1-x} \mathrm{~N}$ active layer was separated from one another by a thin $9 \mathrm{~nm} \mathrm{GaN}$ that was grown at different temperatures. Finally, a $160 \mathrm{~nm}$ thick p-type GaN:Mg cap layer was deposited on the MQWs at $1033^{\circ} \mathrm{C}$. During the growth, the TMIn pressure in the bubbler was fixed at 600 mbar for samples A and B, and 1000 mbar for sample C while the carrier gas content was kept constant for all the samples. The grown structures are shown in figure 1.

X-ray measurements were carried out on a D-8 Bruker high-resolution diffractometer by using $\mathrm{CuK} \alpha_{1}$ $(1.540 \AA$ ) radiation, a prodded mirror, and a 4-bounce Ge (220) symmetric monochromator. The angular resolution of the diffractometer was $0.004^{\circ}$ with the Si calibration sample.

\section{Results and Discussion}

Some structural parameters of the grown samples were identified with the analysis of the data obtained by HRXRD measurements. The recorded $\theta-2 \theta$ X-ray diffraction spectra from the samples are shown in figure 2. The (00.2), (00.4), and (00.6) diffracted peaks for the layers in all the samples were observed. The (00.6) and (00.12) peaks for the sapphire substrate were also observed. These results indicate that all the grown layers on the substrate are mono-crystalline. The $\omega-2 \theta$ profiles for (00.2) and (00.6) symmetric planes for the grown samples are given in figure 3. Moreover, as an example, the $\omega-2 \theta$ profile for the (10.5) asymmetric plane of sample A is shown. As shown in these figures, beside of the InGaN and AlN reflections, an intense (00.2) reflection $\mathrm{GaN}$ and its partial superposition with $\mathrm{InGaN}$ are observed, and the satellite peaks are well resolved, indicating that the GaN, InGaN, and AlN layers have a quality single phase of wurtzite structures. In all curves 


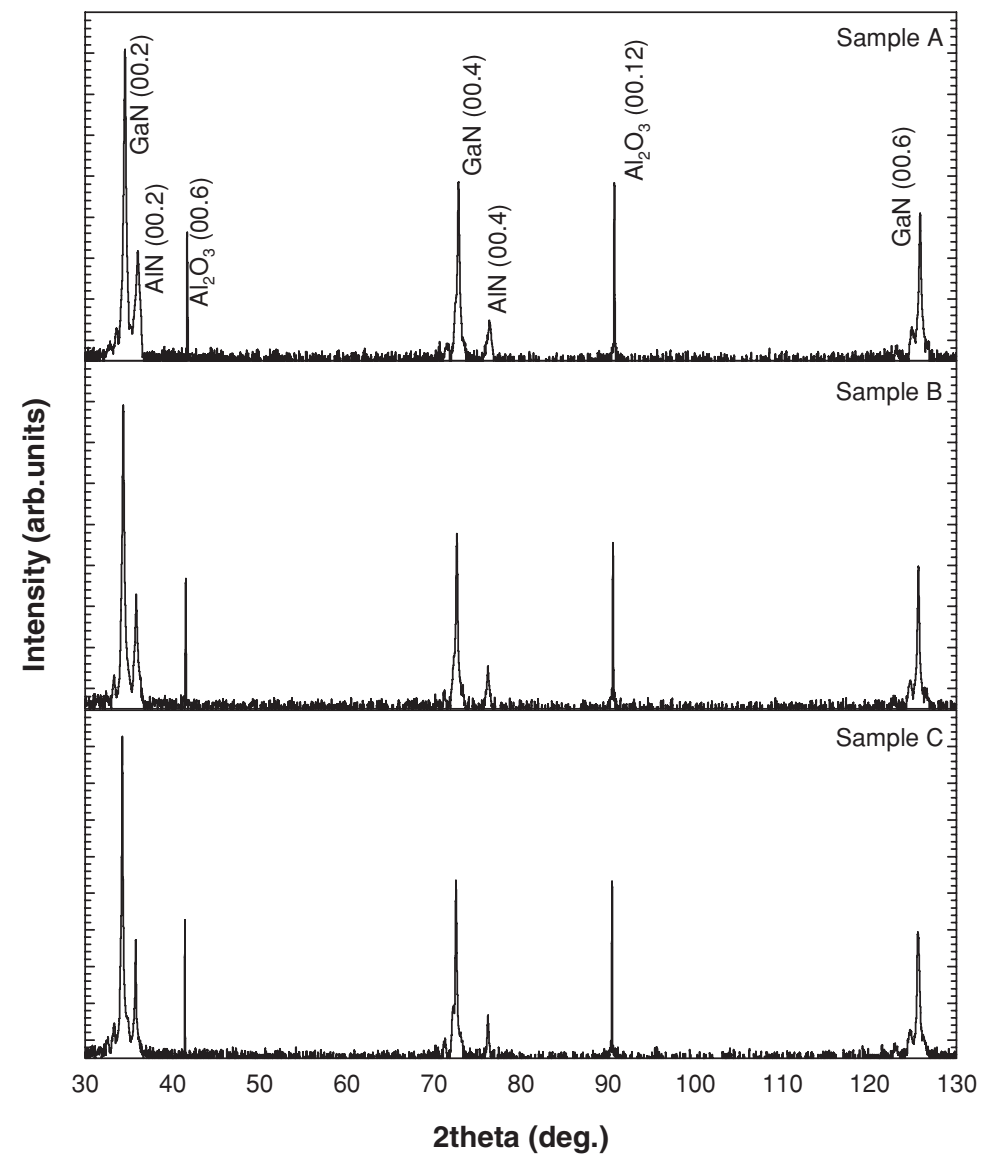

Fig. 2 Recorded the $\theta-2 \theta$ X-ray diffraction spectra for three InGaN/GaN MQW.

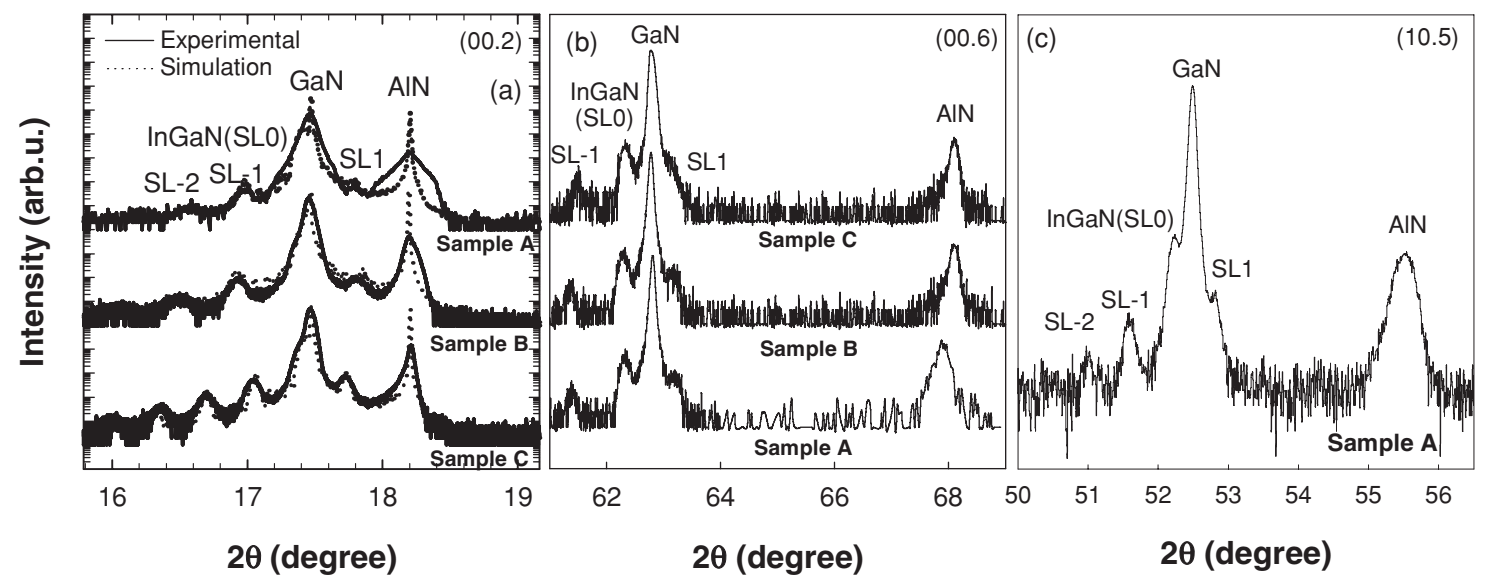

Fig. 3 High-resolution Bragg reflections for symmetric and asymmetric planes (a) (00.2), (b) (00.6) reflection of samples $\mathrm{A}, \mathrm{B}$, and $\mathrm{C}$ and (c) (10.5) reflection of sample A.

in the figure 3a, the reflection peak of the GaN layer, which corresponds to the Bragg reflection from the (00.2) plane of stoichiometric GaN, was observed. The zero-order superlattice peak (SLO) is situated on the left of the peak of GaN layer. The SL0 peaks which are the centers of the pattern were not resolved from the GaN peak at the (00.2) symmetric plane which has higher peak intensities. However, these peaks of the three samples were well separated from the GaN peak both at the (00.6) symmetric and the (10.5) asymmetric plane reflections. The 
path difference between the incident and reflected beams for two planes are different from each other due to the distance between (00.2) and (00.6) planes. With other words, the angle of the incident beam on (00.6) is bigger than the one on (00.2). As the result of these, the peak separation is more evident in (00.6).

Precise determination of the alloy composition of strained heterostructure QW stack is significant to determine the elastic constants (and also Poisson ratio) by excluding the strain effects [23]. It is well known that the composition and other mismatch component of the relaxed layer are determined by the peak separation angle between the Bragg peaks of the layer and the substrate obtained by XRD rocking curve. However, the calculation of alloy content in the strained and partially relaxed layer using XRD data is more complex than fully relaxed cases. For the strained layer, the composition and the layer thickness can be obtained precisely by the evaluation of the XRD measurements [15,24-26]. One specific approach for these evaluations is the approach of Takagi and Taupin [27,28] for deformed epitaxial layers using dynamical X-ray diffraction theory [29]. The definite information about the deformed crystals can be derived from well established simulation procedure using the Takagi-Taupin formulation [26]. However, this formulism is not fully success for analyzing of the partially relaxed layers.

In our grown sample, the In contents in the strained QW have been obtained by using commercial LEPTOS 4.02 software that is based on the solution of the Takagi-Taupin formulation [30]. In addition for this, "LEPTOS has two principal schemes for sample parameter evaluation: (i) empirical estimate and (ii) precise fitting. The former makes an approximate estimation of abovementioned parameters on the basis of substrate/layer peak positions/intensity and interference thickness (Kiessig) fringes. Both kinematical and dynamical approximations are used to evaluate the sample parameters, and analytical expressions provide fast though approximate results [31]. For all the samples, the dotted line (in figures $3 a$ and $b$ ) shows the fit curves of the HRXRD scan using the software. The In contents for the samples A, B, and C have been found as $6.50 \%, 9.75 \%$, and $12.78 \%$, respectively. The alloy composition in the epilayer is very sensitive to the substrate temperature $[32,33]$. The desorption of the In atoms at high growth temperature is stated to be increased due to the decomposition of In-N bond at this temperature [33]. The In incorporation is related to the volatility or desorption of In on the grown surface.

Therefore, at the low growth temperature, volatility/desorption of the In from the surface is reduced and incorporation of In into epi-layer is increased. In contrast, In desorption from the surface is increased by increasing the growth temperature. Accordingly, samples A and B of our study were grown at the same conditions except the growth temperature (at $720^{\circ} \mathrm{C}$ and $710^{\circ} \mathrm{C}$, respectively) and it is found out that the In composition of samples A are smaller than the sample B. On the other hand, sample B and C were grown at the same temperature $\left(710^{\circ} \mathrm{C}\right)$ with different In flow rate. The increase of the In composition in the grown layer could be obtained by increasing In flow rate up to a desired value [33], therefore, In composition in the sample C, which has a greater In flow rate than sample B (figure 1), is higher than sample B.

For the stress-strain analysis, we have used the (00.2), (00.4), (00.6), (10.1), (10.2), (10.5), (11.2), and (12.1) reflections. The out-of-plane, $\varepsilon_{c}$, and in-plane, $\varepsilon_{a}$, strain components of epi-layers can be expressed as

$$
\varepsilon_{c}=\frac{c_{r}-c_{0}}{c_{0}}, \quad \varepsilon_{a}=\frac{a_{r}-a_{0}}{a_{0}}
$$

where $c_{r}$ and $a_{r}$ are strained lattice parameters, and $c_{0}$ and $a_{0}$ are unstrained lattice parameters [14]. The unstrained lattice parameters are $c_{\mathrm{o}}^{\text {GaN }}=5.1855 \AA, c_{\mathrm{o}}^{\operatorname{InN}}=5.7033 \AA, c_{\mathrm{o}}^{A l N}=4.9792 \AA$, and $a_{\mathrm{o}}^{\text {GaN }}=3.1890 \AA$, $a_{\mathrm{o}}^{A l N}=3.1114 \AA, a_{\mathrm{o}}^{I n N}=3.5378 \AA$ for powder GaN, AlN, and InN [34-36]. The $c_{l}$-lattice parameters of $\mathrm{GaN}$, AlN, and InGaN layers have been calculated using the $\omega-2 \theta$-scans of the $(00 . l)$ reflections for $l=$ $2,4,6$;

$$
c_{l}=\frac{l \lambda}{2 \sin \theta_{l}}
$$

where $\theta_{l}$ is the peak position of the (00.l) reflections, and $\lambda$ is the wavelength of the $\mathrm{CuK} \alpha_{1}$ reflection [37].

The precision in the measurement of the lattice constant depends on the precision in $\sin \theta$, as seen in equation 2 . To calculate the ideal lattice parameters, using an extrapolating function can reduce the errors in the measurements of the $\theta$ angle in the XRD scan. The error of the $c_{l}$-lattice parameters can be given as [14,37]

$$
\frac{\Delta c}{c_{r}}=-\frac{D}{r} p_{l}
$$



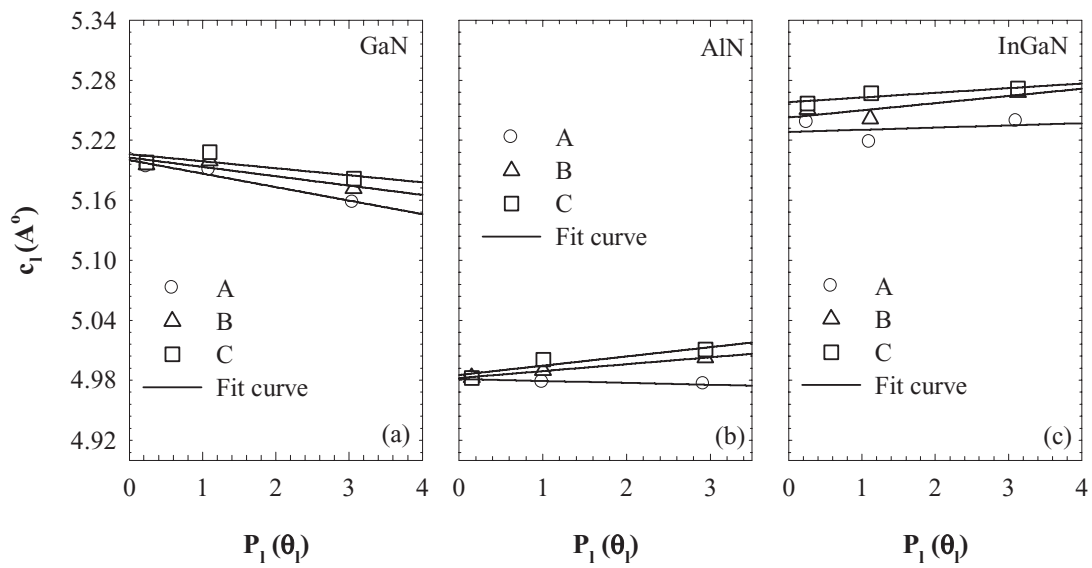

Fig. 4 The extrapolation of the $c_{l}$ lattice parameter of $\mathrm{GaN}$, AlN and $\mathrm{InGaN}$ layer in the samples versus $\cos ^{2} \theta_{l} / \sin \theta_{l}$.

Table 1 Measured c- and a- strained lattice parameters.

\begin{tabular}{lcccc}
\hline Samples & GaN & AlN & InGaN \\
\hline A & $\mathrm{a}(\AA)$ & 3.1816 & 3.0781 & 3.2071 \\
$\mathrm{~B}$ & & 3.1818 & 3.0736 & 3.2132 \\
$\mathrm{C}$ & 3.1818 & 3.0782 & 3.2212 \\
$\mathrm{~A}$ & $\mathrm{c}(\AA)$ & 5.2001 & 4.9812 & 5.2282 \\
$\mathrm{~B}$ & & 5.2027 & 4.9822 & 5.2427 \\
$\mathrm{C}$ & 5.2060 & 4.9853 & 5.2580 \\
\hline
\end{tabular}

where $\Delta c=c_{l}-c_{r}$ and $p_{l}$ is an extrapolation function given by

$$
p_{l}\left(\theta_{l}\right)=\frac{\cos ^{2} \theta_{l}}{\sin \theta_{l}}
$$

where $\theta_{l}$ is the peak position of the $(h k . l)$ reflection, $r(\sim 415 \mathrm{~mm})$ is the distance specimen detector, $D\left(\sim 0.145^{\circ}\right)$ is the possible displacement of the specimen with respect to the goniometer axis in the equatorial plane and the lattice parameter $c_{r}$ is determined from the plots $\left\{p_{l}\left(\theta_{l}\right), c_{l}\right\},(l=2,4,6)$ [37]. The extrapolation of the $c_{l}$ lattice parameter of the GaN, AlN, and InGaN layers in samples A, B, and C is illustrated in figure 4. The true value of the lattice parameter $(c)$ was obtained by this extrapolating linear line of the lattice parameter versus the extrapolation function given in equation (4). The obtained strained $c$-lattice parameters $\left(c=c_{r}\right)$ for the GaN, AlN, and InGaN hexagonal system are shown in table 1.

The $a$-lattice parameter of the layers for the diffraction peaks of the $(h k . l)$ asymmetrical reflections is found by the below equation [37];

$$
a^{(h k l)}=c d_{h k l} \sqrt{\frac{(4 / 3)\left(h^{2}+k^{2}+h k\right)}{c^{2}-l^{2} d_{h k l}^{2}}}
$$

where $d_{h k l}$ is the distance of the interplanes. Using the above mentioned extrapolation method, the calculated $a$-strained values for GaN, AlN, and InGaN hexagonal structures are given in table 1 .

The unstrained $a$ - and $c$-lattice parameters for $\operatorname{In}_{x} \mathrm{Ga}_{1-x} \mathrm{~N}$ layers for all the samples were calculated by using Vegard's law; $a_{I n_{x} G a_{1-x} N}=x a_{I n N}+(1-x) a_{G a N}[38,39]$ with the unstrained lattice constants of GaN and InN in powder form [34-36] and using the In composition which obtained from the evaluation XRD data for the grown samples. Here x, unstrained $a_{I n N}$ and unstrained $a_{G a N}$ is known. Unstrained $a_{I n_{x} G a_{1-x} N}$ are used to obtain strain properties. The obtained lattice parameters are given in table 2. In addition, the ideal ratio of lattice parameters (c/a) for an ideal hexagonal crystal is 1.633 [23]. However, it is well known that the c/a-ratios of wurtzite ternary 
Table 2 Calculated unstrained c- and a-lattice parameters of the InGaN epilayer using Vegard's law.

\begin{tabular}{lccc}
\hline & \multicolumn{3}{c}{ Sample } \\
\cline { 2 - 4 } Parameters & $\mathrm{A}$ & $\mathrm{B}$ & $\mathrm{C}$ \\
\hline $\mathrm{a}(\AA)$ & 3.2117 & 3.2231 & 3.2336 \\
$\mathrm{c}(\AA)$ & 5.2192 & 5.2360 & 5.2517 \\
\hline
\end{tabular}

GaN-based alloys are below this value. For samples A, B, and C the $c / a$ ratio of $\mathrm{InGaN}$ was calculated as 1.630, 1.632 , and 1.632 , respectively.

The calculated normal strains, depending on In content of InGaN in the $a$-and $c$-directions for all epitaxial layers of three LED structures, are shown in figures $5 \mathrm{a}$ and $\mathrm{b}$. In all the epilayers, the calculated elastic strains in the $a$ - and $c$-directions can be of the compressive (negative strain) and tensile (positive strain) types, respectively, which strongly exhibited characteristic behavior. In the $a$-directed and $c$-directed strains of the InGaN and the thick GaN and AlN buffer in the samples are the compressive and tensile types, respectively. In these two directions, the strain magnitudes in the AlN layers are larger than the GaN layers. The strains in the $c$-direction for these layers show similar behavior. These cases are related with the presence of their deformation states coming from the large difference between the lattice parameters or lattice mismatches between AlN and GaN. As seen in figures $5 \mathrm{a}$ and $\mathrm{b}$, the strain in the c-direction of the InGaN layer is decreased with the increasing of the In content while the strain in the $a$-direction of the InGaN layer is increased with the In content. The strains of the InGaN layers were the compressive type in the $a$-directions when the $c$-directed strains of the samples were tensile. Strains in different directions have to be in different behaviors as tensile or compressive due to the in-plane and out of plane lattice mismatch. Difference in the lattice constants and thermal expansion coefficient between the grown layers leads to formation of deformation in the heterostructures [40]. The deformation state in the InGaN was affected from the behaviors of the GaN and AlN layers as shown in figures $5 \mathrm{a}$ and $\mathrm{b}$. Here, it is well known that the AlN layer plays an important role in the reduction of the mismatch between GaN and Sapphire. Moreover, the deformation states in the InGaN epilayers have appeared with point defects that originated from the large difference in the covalent radii of the Ga, N, Al, and In atoms $\left(r_{G a}=0.126 \mathrm{~nm}, r_{N}=0.07 \mathrm{~nm}, r_{A l}\right.$ $=0.147 \mathrm{~nm}, r_{I n}=0.144 \mathrm{~nm}$ ). Furthermore, the grown layers can be affected by common impurities or doping materials (oxygen, elements to induce $n$ or p-type carriers).

By hydrostatic pressure coming from point defects, lattice parameters widens as volume at equal values. But by biaxial stress, the lattice parameters widen in plane at equal values. The stresses in the heteroepitaxial films are biaxial by nature. It is established that in $\mathrm{GaN} / \mathrm{sapphire}$ heterostructures the character of the stress originating from the mismatch between the epilayer and the substrate lattice parameters is really biaxial $[10,11,41,42]$.

The obtained values of $\varepsilon_{c}$ and $\varepsilon_{a}$ are superpositions of the biaxial strain components $\varepsilon_{c}^{b}$ and $\varepsilon_{a}^{b}$, and of the hydrostatic strain component $\varepsilon_{h}[10,11,14]$;

$$
\varepsilon_{c}=\varepsilon_{c}^{b}+\varepsilon_{h}, \quad \varepsilon_{a}=\varepsilon_{a}^{b}+\varepsilon_{h}
$$

where $\varepsilon_{h}$ is given by

$$
\varepsilon_{h}=\frac{1-v}{1+v}\left(\varepsilon_{c}+\frac{2 v}{1-v} \varepsilon_{a}\right)
$$

The hydrostatic strain $\varepsilon_{h}$ depends on the Poisson ratio, which is determined from the elastic constants $\mathrm{C}_{13}$ and $\mathrm{C}_{33}$ with the relation of $v=c_{13} /\left(c_{13}+c_{33}\right)$. The elastic constants of GaN [43], AlN [44], and InN [45] are given in table 3 with the calculated Poisson ratio. In addition, the elastic constants and Poisson ratio of InGaN layers for all the grown samples have been calculated using the $a$ - and $c$ - lattice parameters obtained from Vegard's law $[46,47]$. The calculated values are presented in table 4.

Using the above relations, the calculated biaxial strain components of the GaN, AlN, and InGaN layers are shown in figures $5 \mathrm{c}$ and $\mathrm{d}$. These components for the InGaN, GaN, and AlN layers in the $a$ - and $c$ - directions in the all the samples were of the compressive and tensile types, respectively. As seen in these figures, the biaxial strain for the InGaN layer was increased with the In content in two directions. If the hydrostatic strain is zero, the Poisson ratio (or elastic constants) is related to the ratio of the strain components $\varepsilon_{c} / \varepsilon_{a}=-2 v /(1-v)[10,17]$. In the presence of the hydrostatic strain, we calculated the strain ratio using the extracted values from the $\varepsilon_{h}$ of 

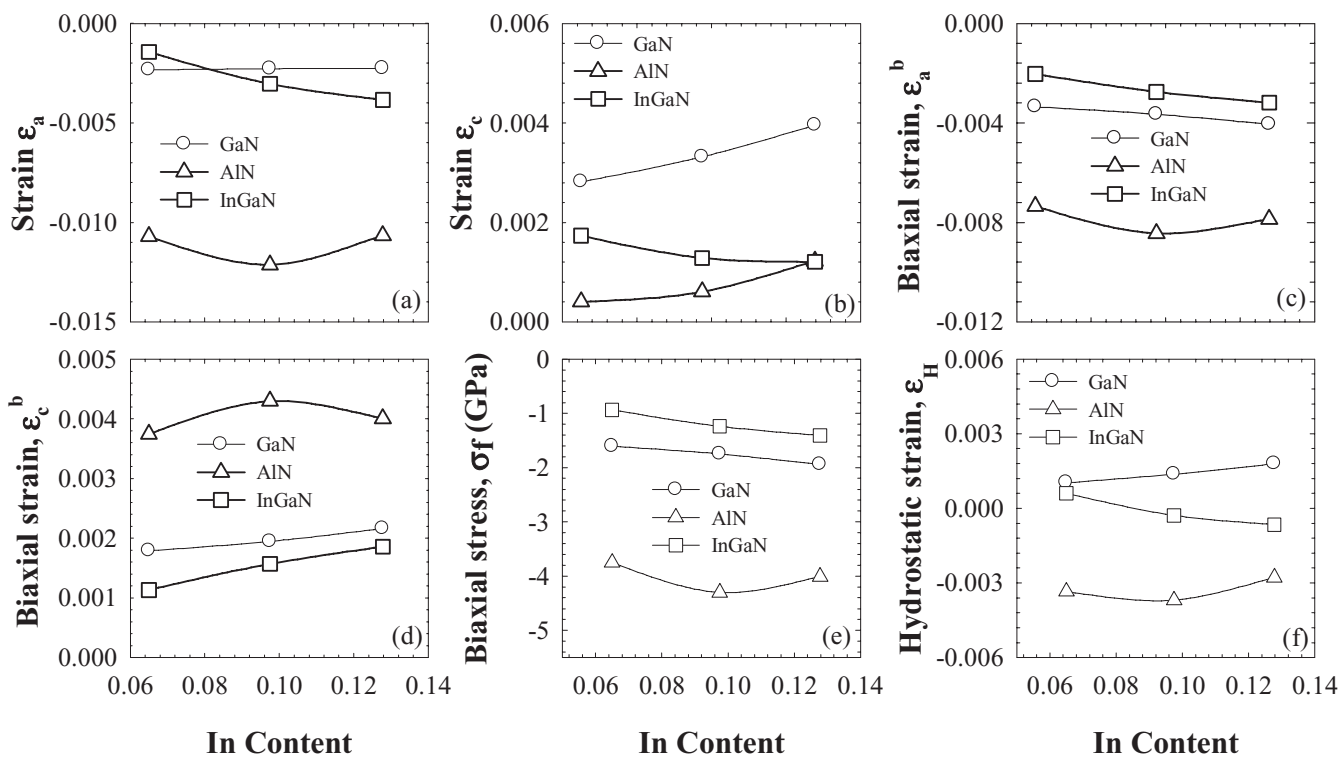

Fig. 5 The calculated strains in the (a) $a$ - and (b) $c$-directions versus the In content of GaN, AlN, and InGaN structures for three samples. The biaxial strain components versus the In content of GaN, AlN, and InGaN structures for three samples: (c) $a$ - direction and (d) $c$-direction. (e) The biaxial stress versus the In content of $\mathrm{GaN}, \mathrm{AlN}$, and InGaN structures for three samples. (f) The hydrostatic strain versus the In content of the GaN, $\mathrm{AlN}$, and InGaN structures for three samples.

Table 3 The elastic constants and calculated Poisson ratio for GaN, AlN, and InN.

\begin{tabular}{lccc}
\hline Parameters & GaN & AlN & InN \\
\hline $\mathrm{C} 11(\mathrm{GPa})$ & $390 \pm 15^{a}$ & $411 \pm 10^{b}$ & $190 \pm 7^{c}$ \\
$\mathrm{C} 12(\mathrm{GPa})$ & $145 \pm 20^{a}$ & $149 \pm 10^{b}$ & $104 \pm 3^{c}$ \\
$\mathrm{C} 13(\mathrm{GPa})$ & $106 \pm 20^{a}$ & $99 \pm 4^{b}$ & $121 \pm 7^{c}$ \\
$\mathrm{C} 33(\mathrm{GPa})$ & $398 \pm 20^{a}$ & $389 \pm 10^{b}$ & $182 \pm 6^{c}$ \\
$\mathrm{M}_{\mathrm{f}}(\mathrm{GPa})$ & $478.5 \pm 16$ & $509.6 \pm 17$ & $133.1 \pm 3$ \\
Poisson ratio & $0.210 \pm 0.023$ & $0.203 \pm 0.002$ & $0.399 \pm 0.005$ \\
\hline
\end{tabular}

${ }^{a}[43],{ }^{b}[44],{ }^{c}[45]$.

Table 4 Calculated elastic constants and Poisson ratios for the InGaN epilayer.

\begin{tabular}{lccc}
\hline & & InGaN & \\
Parameters & Sample A & Sample B & Sample C \\
\hline C11 $(\mathrm{GPa})$ & 377.0 & 370.5 & 364.4 \\
$\mathrm{C} 12(\mathrm{GPa})$ & 142.3 & 141.0 & 139.8 \\
$\mathrm{C} 13(\mathrm{GPa})$ & 107.0 & 107.5 & 107.9 \\
$\mathrm{C} 33(\mathrm{GPa})$ & 384.0 & 376.9 & 370.4 \\
$\mathrm{M}_{\mathrm{f}}(\mathrm{GPa})$ & 459.7 & 450.2 & 441.3 \\
Poisson ratio & $0.218 \pm 0.022$ & $0.222 \pm 0.022$ & $0.226 \pm 0.021$ \\
\hline
\end{tabular}

the biaxial strain $\left.\varepsilon_{c}^{b} / \varepsilon_{a}^{b}=-2 v /(1-v)\right)$. This ratio for samples $\mathrm{A}, \mathrm{B}$, and $\mathrm{C}$ was found to be $-0.56,-0.57$, and -0.58 , respectively. These results are in agreement with the literature [10,17,23]. In addition, the strain ratio increased with increasing In content in the samples. However, the strain ratio, $\varepsilon_{\mathrm{c}} / \varepsilon_{\mathrm{a}}$, depends on the amount of biaxial stress, the concentration of point defects, and the ratio of the atomic covalent radii of the species in the layer [17]. 
Biaxial strains come from the growth on the lattice-mismatched sapphire substrate and post-growth cooling [13]. This behaviour is evaluated from the thermal strains $\left(\varepsilon_{\text {thermal }}\right)$. $\varepsilon_{\text {thermal }}$ can be calculated from the thermal expansion coefficient TEC $(\alpha)$, which is derived from the post-growth cooling from the growth temperature to room temperature using the relation

$$
\varepsilon_{\text {thermal }}=\int_{T_{\text {room }}}^{T_{\text {growth }}}\left(\alpha_{\text {layer }}-\alpha_{\text {substrate }}\right) d T
$$

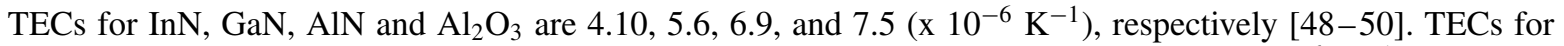

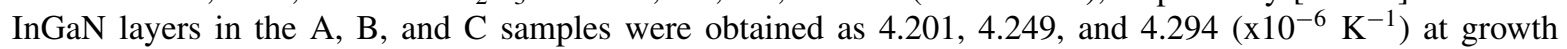
temperature by assuming that the TECs of the InGaN change linearly with In content. As expected, the TEC of InGaN has changed between the TECs of AlN and GaN. The thermal strains of the grown GaN layer on the AlN and the grown AlN layer on $\mathrm{Al}_{2} \mathrm{O}_{3}$ were calculated as -1.379 and $-0.488\left(\times 10^{-3}\right)$, respectively. The thermal strain of the grown $\mathrm{GaN}$ on sapphire is $-2.014 \times 10^{-3}$ [13]. These results indicate that the compressive strain in the structures was reduced by growing AIN on the sapphire, as expected. The thermal strain of the grown InGaN layer on the GaN layer in the A, B, and C samples was also calculated as $-0.963,-0.916$, and $-0.885\left(\mathrm{x} 10^{-3}\right)$, respectively. The magnitude of $\varepsilon_{\text {thermal }}$ in InGaN for all the samples has decreased by increasing the In content in the layer. At the same time, the thermal strains values with an order of approx. $10^{-1}$ in all the layers are smaller than the biaxial strains for all the layers. The biaxial strains may be affected by some dislocations generated during the cooling process.

The in-plane biaxial stress in the epilayer $\sigma_{\mathrm{f}}$ can be calculated from the relationship:

$$
\sigma_{f}=\left(c_{11}+c_{12}-2 \frac{c_{13}^{2}}{c_{33}}\right) \varepsilon_{a}^{b}
$$

where the inside of the parentheses the biaxial elastic modulus is labelled with $M_{f}$ and, therefore, biaxial stress is demonstrated as $\sigma_{f}=M_{\mathrm{f}} \varepsilon_{a}^{b}$ [14]. The biaxial stress in the layers of the samples is calculated and shown in figure 5e. As seen in this figure, the biaxial stress of all the layers shows compressive behaviour. The stress in the layers with increasing In contents for the samples shows characteristic behaviour. As the In content is increased, the biaxial stress of the InGaN layers was also increased in this direction.

The hydrostatic strain is affected by doping and point defects in the alloy structures [10]. This strain can be compressive or expansive depending on the size of the defects. The various type point defects can be formed depending on the growth conditions such as substitutional type point defects $\left(\mathrm{Al}_{G a}, \mathrm{Ga}_{N}, \mathrm{~N}_{I n}, \mathrm{Al}_{N}\right.$, and $\left.\mathrm{Ga}_{I n}\right)$, interstitial point defects $\left(\mathrm{N}_{\mathrm{i}}, \mathrm{Ga}_{\mathrm{i}}, \mathrm{Al}_{\mathrm{i}}\right.$, and $\left.\mathrm{In}_{\mathrm{i}}\right)$, and vacancies $\left(\mathrm{V}_{A l}, \mathrm{~V}_{N}, \mathrm{~V}_{I n}\right.$, and $\left.\mathrm{V}_{G a}\right)$ due to the covalent radii of the $\mathrm{Al}, \mathrm{Ga}$, and In atoms being considerably larger than the covalent radius of the $\mathrm{N}$ atom. Therefore, the $\mathrm{Al}_{G a}$, $\mathrm{Ga}_{N}, \mathrm{In}_{N}, \mathrm{Al}_{N}, \mathrm{In}_{G a}, \mathrm{~N}_{\mathrm{i}}, \mathrm{Ga}_{\mathrm{i}}, \mathrm{Al}_{\mathrm{i}}$ and $\mathrm{N}_{\mathrm{i}}$ type defects also cause a crystal expansion, whereas $\mathrm{Ga}_{A l}, \mathrm{~N}_{G a}, \mathrm{~N}_{I n}, \mathrm{~N}_{A l}$, $\mathrm{Ga}_{I n}, \mathrm{~V}_{A l}, \mathrm{~V}_{G a}, \mathrm{~V}_{I n}$, and $\mathrm{V}_{N}$ type point defects lead to crystal compression as described in [10,13]. The hydrostatic strains for all the layers in the grown samples were calculated by using equation 7 . The error introduced in the value of $\varepsilon_{h}$ for AlN, GaN, and InGaN layers in samples A, B, and C by the uncertainty in the measured values of $\mathrm{C}_{13}$ and $\mathrm{C}_{33}$ were estimated as $0.00007,0.00004$, and 0.0002 , respectively. The calculated hydrostatic strains, for the AlN, GaN, and InGaN layers in all the samples are given in figure $5 f$. As can be seen in this figure, in case of the sample A the hydrostatic strain in the InGaN QW layer was tensile while the hydrostatic strain of samples B and $\mathrm{C}$ were compressive. With other words, $\mathrm{N}_{G a}, \mathrm{~N}_{I n}, \mathrm{Ga}_{I n}, \mathrm{~V}_{G a}, \mathrm{~V}_{I n}$, and $\mathrm{V}_{N}$ type point defects are dominant for samples $\mathrm{B}$ and $\mathrm{C}$, whereas, $\mathrm{Ga}_{N}, \mathrm{In}_{N}, \mathrm{In}_{G a}, \mathrm{In}_{\mathrm{i}} \mathrm{Ga}_{\mathrm{i}}$, and $\mathrm{N}_{\mathrm{i}}$ type point defects are dominant for sample $\mathrm{A}$. This type of defects or the tensile type hydrostatic strain in sample A may be attributed to increasing of interstitial point defects. The compressive hydrostatic strains suggest that the GaN and AlN have the substitutional and interstitial type point defects. Additionally, the magnitude of the hydrostatic strain of the InGaN QW layer is smaller than the magnitude of $\varepsilon_{h}$ for the GaN layer in all the samples. This may be explained with low point defect of the InGaN with respect to $\mathrm{GaN}$ layers since hydrostatic strain in $\mathrm{GaN}$ is affected by doping and point defects [10]. In addition, since the hydrostatic strain was smaller than $\varepsilon_{a}$, therefore, $\varepsilon_{a}^{b}$ is slightly different than $\varepsilon_{a}$. 


\section{Conclusion}

In this work, five period three quantum well structure were grown by MOVD and the strain and stress analysis of the structures were studied by evaluation of obtained HRXRD data. The biaxial and hydrostatic components were extracted from the strain values obtained, and were then discussed in the present study as the effect of the increasing In content depending on growth conditions in $\operatorname{In}_{x} \mathrm{Ga}_{1-x} \mathrm{~N}$. The normal strain components and biaxial strains in the $a$-direction showed a little decreasing with the In content. At the same time, the normal and biaxial strains in the $a$-direction showed the opposite behaviour of the ones in the $c$-direction. The normal strains in the every two directions for InGaN showed a decrease with a small positive bowing. On the other hand, the $a$ - and $c$-biaxial strains of the InGaN behaved compressive and tensile, respectively. The reason for this behaviour is from the post growth cooling, lattice and thermal mismatch. The compressive hydrostatic strains suggest that the related layers have the substitutional and vacancy type point defects while the tensile strained layers have the substitutional and interstitial type point defects.

Acknowledgments This work was supported by DPT under the project no. 2011K120290 and by BAP at Gazi Univ. under project no. 05/2009-58.

\section{References}

[1] S. Nakamura, M. Senoh, S. Nagahama, N. Iwasa, T. Matsushita, and T. Mukai, Appl. Phys. Lett. 76, 22 (2000).

[2] I. Akasaki and H. Amano, Jpn. J. Appl. Phys. 1, 5393 (1997).

[3] W. T. Liaoa, J. R. Gong, S. W. Lina, C. L. Wanga, T. Y. Lin, K. C. Chena, Y. C. Chengd, and W. J. Lin, J. Cryst. Growth 286, 28 (2006).

[4] High-Brightness LED Market Review and Forecast 2007, Published: Strategies Unlimited (2007).

[5] J. H. Ryou, W. Lee, J. Limb, D. Yoo, J. P. Liu, R. D. Dupuis, Z. H. Wu, A. M. Fischer, and F. A. Ponce, Appl. Phys. Lett. 92, 1 (2008).

[6] A. Yıldız, M. K. Öztürk, M. Bosi, S. Ozcelik, and M. Kasap, Chinese Phys. B 18, 4007 (2009).

[7] A. Krost, A. Dadgar, G. Strassburger, and R. Clos, Phys. Stat. Sol. (a) 200, 26 (2003).

[8] M. K. Ozturk, Y. Hongbo, B. Sarikavak, S. Korcak, S. Ozcelik, and E. Ozbay, J. Mater. Sci:Mater. Electron. 21, 185 (2010).

[9] M. K. Ozturk, H. Altuntas, S. Corekci, Y. Hongbo, S. Ozcelik, and E. Ozbay, Strain 47, 19 (2010).

[10] C. Kisielowski, J. Kruger, S. Ruvimov, T. Suski, J. W. Ager, E. Jones, Z. Liliental-Weber, M. Rubin, E. R. Weber, M. D. Bremser, and R. F. Davis, Phys. Rev. B 54, 17745 (1996).

[11] C. Kisielowski and Semicon. Semimet. 57, 275 (1999).

[12] S. I. Cho, K. Chang, and M. S. Kwon, J. Mater. Sci. 43, 406 (2008).

[13] S. I. Cho, K. Chang, and M. S. Kwon, J. Mater. Sci. 42, 3569 (2007).

[14] V. S. Harutyunyan, A. P. Aivazyan, E. R. Weber, Y. Kim, Y. Park, and S. G. Subramanya, J. Phys. D: Appl. Phys. 34, A35 (2001).

[15] M. A. G. Halliwell, J. Cryst. Growth, 170, 47 (1997).

[16] M. Leszczynskia, P. Prystawko, T. Suski, B. Lucznik, J. Domagala, J. Bak-Misiuk, A. Stonert, A. Turos, R. Langer, and A. Barski, J. Alloy. Compd. 286, 271 (1999).

[17] E. Z. Rohozinska, J. Gronkowskia, K. PakulÇaa, M. Majera, M. Regulskaa, and L. Nowicki, J. Alloy. Compd. 328,199 (2001).

[18] H. Morkoc, Nitride Semiconductors and Devices (Springer-Verlag, Berlin, Heidelberg, New York, 1999).

[19] C. Roder, S. Einfeldt, S. Figge, T. Paskova, D. Hommel, P. P. Paskov, B. Monemar, U. Behn, and B. A. Haskell, P. T. Fini, S. Nakamura, J. Appl. Phys. 100, 1 (2006).

[20] S. Hearne, E. Chason, J. Han, J. A. Floro, J. Figiel, J. Hunter, H. Amano, and T. Song, Appl. Phys. Lett. 74, 356 (1999).

[21] S. Raghavan, J. Acord, and J. M. Redwing, Appl. Phys. Lett. 86, 1 (2005).

[22] T. Bottcher, S. Einfeldt, S. Figge, R. Chierchia, H. Heinke, D. Hommel, and J. S. Speck, Appl. Phys. Lett. 78, 1976 (2001).

[23] M. A. Moram and M. E. Vickers, Rep. Prog. Phys. 72, 1 (2009).

[24] C. Ferrari and C. Bocchi, Characterization of Semiconductor Heterostructure and Nanostructure, edited by C. Lamberti and B. V. Elsevier (2008).

[25] A. Benediktovich and I. Feranchuk, Phys. Status Solidi A 206, 1695 (2009).

[26] Y. Epelboin, Mater. Sci. and Engineering, 73, 1 (1985).

[27] S. Takagi, Acta Crystallogr. 15, 1311 (1962).

[28] D. Taupin, Bull. Soc. Fr. Mindral. Crystallogr. 57, 469 (1964). 
[29] A. Authier, Dynamical Theory of X-ray Diffraction, in: ICUR Monographs on Crystallography Vol. 11 (Oxford University Press Inc., New York, 2001).

[30] LEPTOS User Manual (www.bruker-axs.de), Version 2 (2004).

[31] A. Ulyanenkov, Applied Surface Science 253, 106 (2006).

[32] N. Yoshimoto, T. Matsuoka, T. Sasaki, and A. Katsui, Appl. Phys. Lett. 59, 2251 (1991).

[33] Y. Guo, X. L. Liu, H. P. Song, A. L. Yang, X. Q. Xu, G. L. Zheng, H. Y. Wei, S. Y. Yang, Q. S. Zhu, and Z. G. Wang, Appl. Surf. Sci. 256, 3352 (2010).

[34] C. M. Balkas, C. Basceri, and R. Davis, Powder Diffraction 10, 266 (1995).

[35] National Bureau of Standards (US), Monograph No. 25, 1975, p. 125 (cited in the PDF files \#25).

[36] W. Paszkowicz, ICDD Grant-in-Aid, Polish Academy of Sciences (Warsaw, Poland, 1998).

[37] C. Suryanarayana and M. G. Norton, X-Ray Diffraction: A practical Approach (Plenum Press, New York, 1998).

[38] R. Singh, D. Doppalapudi, T. D. Moustakas, and L. T. Romano, Appl. Phys. Lett. 70, 1089 (1997).

[39] F. K. Yam and Z. Hassan, Superlattice. Microst. 43, 1 (2008).

[40] S. A. Kukushkin, A. V. Osipov, V. N. Bessolov, B. K. Medvedev, V. K. Nevolin, and K. A. Tcarik, Rev. Adv. Mater. Sci. 17, 1 (2008).

[41] W. G. Perry, T. Zheleva, M. D. Bremser, R. F. Davis, W. Shan, and J. J. Song, J. Electron. Mater. 26, 224 (1997).

[42] B. J. Skromme, H. Zhao, D. Wang, H. S. Kong, M. T. Leonard, G. E. Bulman, and R. J. Molnar, Appl. Phys. Lett. 71, 829 (1997).

[43] A. Polian, M. Grimsditch, and I. Grzegory, J. Appl. Phys. 79, 3343 (1996).

[44] L. E. McNeil, M. Grimsditch, and R. H. French, J. Am. Ceram. Soc. 76, 1132 (1993).

[45] A. U. Sheleg and V. A. Savastenko, Izv. Akad. Nauk SSSR, Neorg. Mater. 15, 1598 (1979).

[46] F. M. Morales, D. Gonzalez, J. G. Lozano, R. Garcia, S. Hauguth-Frank, V. Lebedev, V. Cimalla, and O. Ambacher, Acta Materialia 57, 5681 (2009).

[47] M. E. Vickers, M. J. Kappers, T. M. Smeeton, E. J. Thrush, J. S. Barnard, and C. J. Humphreys, J. Appl. Phys. 94, 1565 (2003).

[48] K. Wang and R. R. Reeber, Appl. Phys. Lett. 79, 1602 (2001).

[49] I. Ahmad, M. Holtz, N. N. Faleev, and H. Temkin, J. Appl. Phys. 95, 1692 (2004).

[50] R. R. Reeber and K. Wang, MRS Internet J. Nitride Semicond. Res. 6, 1 (2001). 